\title{
Índices antropométricos de crianças assistidas em creches e sua relação com fatores socioeconômicos, maternos e infantis
}

\author{
Anthropometric indices of children treated in daycare centers \\ and the relationship with socioeconomic, maternal and child factors
}

Dixis Figueroa Pedraza ${ }^{1}$

Maria Monica de Oliveira ${ }^{2}$

Maria Vera Lúcia Moreira Leitão Cardoso ${ }^{3}$

Erika Morganna Neves de Araujo ${ }^{1}$

Ana Carolina Dantas Rocha ${ }^{3}$

${ }^{1}$ Programa de Pós-

Graduação em Saúde Pública, Departamento de Enfermagem, Universidade Estadual da Paraíba. Av. das Baraúnas 351, Bodocongó 58109-753 Campina Grande PB Brasil. dixisfigueroa@gmail.com ${ }^{2}$ Instituto Educacional Particular Brasileiro Carúbas PB Brasil. ${ }^{3}$ Departamento de Enfermagem, Universidade Federal do Ceará. Fortaleza CE Brasil.

\begin{abstract}
Weight/height and weight/age anthropometric statistics are important, respectively, for identifying incipient changes in weight and alterations in nutritional status at an early stage. The scope of this study was to analyze weight/heightand weight/age-associated factors in preschool children. This is a cross-sectional study with a probabilistic sample scrutinizing 299 children enrolled in municipal daycare centers. Information was obtained through interviews with mothers together with a questionnaire analyzing socioeconomic, maternal and child information. Weight and height/stature of children were measured according to recommendations of the World Health Organization. The results showed that none of the maternal variables was associated with the nutritional status of children and that only birth weight among child variables revealed any association with weight/height $(p=0.0030)$ and weight/age scores $(p=0.0018)$. The block of socioeconomic variables, in turn, proved to be the most representative variation factor of results, especially in the weight/age index. The importance of birth weight in the weight/height and weight/age of children, as well as the socioeconomic conditions notably in weight/age indices, was clearly revealed.
\end{abstract}

Key words Nutritional status, Preschool, Daycare centers
Resumo Os indices antropométricos peso/estatura e peso/idade destacam-se, respectivamente, por sua importância na identificação de mudanças recentes de peso e de alterações no estado nutricional de forma precoce. O objetivo deste estudo foi analisar fatores associados ao peso/estatura e peso/idade em crianças pré-escolares. Trata-se de um estudo transversal com amostra probabilística que analisou 299 crianças assistidas em creches públicas municipais. As informações foram obtidas por meio de entrevista com as mães, mediante aplicação de um questionário com informações socioeconômicas, maternas e infantis. O peso e a estatura/comprimento das crianças foram medidos seguindo as recomendações da Organização Mundial de Saúde. Os resultados evidenciaram que nenhuma das variáveis maternas associou-se ao estado nutricional das crianças e que apenas o peso ao nascer, dentre as variáveis infantis, apresentou associação aos escores de peso/estatura ( $p=$ $0,0030)$ e peso/idade $(p=0,0018)$. O bloco de variáveis socioeconômicas, por sua vez, mostrou-se como o mais representativo fator de variação dos resultados encontrados, sobretudo no peso/idade. Conclui-se sobre a relevância do peso ao nascer no peso/estatura e peso/idade das crianças, bem como das condições socioeconômicas, marcadamente, no peso/idade.

Palavras-chave Estado nutricional, Pré-escolar, Creches 


\section{Introdução}

A antropometria é importante para estimar as prevalências e a gravidade de alterações nutricionais ${ }^{1}$. As medidas antropométricas mais utilizadas para avaliar e monitorar o estado nutricional das crianças são o peso e a estatura ${ }^{2}$. Para uma maior acurácia do diagnóstico nutricional recomenda-se o uso de medidas antropométricas combinadas ${ }^{3}$. Assim, os índices antropométricos têm sido considerados válidos para gerar indicadores sensíveis do estado nutricional ${ }^{4}$.

Além de a desnutrição crônica continuar sendo a expressão epidemiológica mais característica e representativa de desvio antropométrico da população infantil no Brasil $^{5}$ e na região da América Latina ${ }^{6}$, há evidências empíricas sobre a magnitude dos impactos do déficit de peso em termos de morbimortalidade e aprendizado ${ }^{6}$, e sobre o aumento do excesso de peso que configura um problema emergente de saúde pública no mundo $^{7}$ e no Brasil ${ }^{8}$. Para o monitoramento desses problemas, o peso/idade torna possível identificar alterações no estado nutricional precocemente e o peso/estatura é indicado para avaliar alterações recentes de peso, inclusive de excesso ${ }^{1}$.

O diagnóstico do déficit de peso (estabelecido pelo peso/idade) possibilita maior comparabilidade entre países ${ }^{6}$, é o indicador nutricional utilizado para medir alcances relacionados aos Objetivos de Desenvolvimento do Milênio9 e constitui o indicador mais visível do problema nutricional nos primeiros anos da vida. Por isso é usado mundialmente em puericultura e recomendado na avaliação de impacto de intervenções nutricionais ${ }^{1,6}$.

O desenvolvimento do sobrepeso e da obesidade, por sua vez, tem sido constatado em idades cada vez mais precoces, especialmente em pré-escolares $^{10}$. O excesso de peso na infância constitui um fator de risco da obesidade na idade adulta e do desenvolvimento de doenças crônicas, além de acarretar isolamento do convívio social e discriminação causando grande impacto na qualidade de vida ${ }^{11}$. Quando diagnosticado ainda na infância, o tratamento do sobrepeso e da obesidade pode ser mais eficiente e menos oneroso comparado a intervenções em adultos, que têm se mostrado pouco eficazes ${ }^{12}$.

Com esta justificativa, e considerando, ain$\mathrm{da}$, que o conhecimento dos determinantes do estado nutricional de crianças constitui uma ferramenta indispensável na prevenção e detecção precoce de distúrbios nutricionais, o presente estudo tem como objetivo analisar fatores asso- ciados ao peso/estatura e peso/idade em crianças pré-escolares.

\section{Métodos}

Trata-se de um estudo transversal, integrado ao projeto "Saúde e nutrição das crianças assistidas em creches públicas do município de Campina Grande, Paraíba". A coleta de dados contou com a participação de uma equipe treinada, composta por professores e alunos de graduação da área de saúde ou de correlatas, no período de outubro a novembro de 2011.

O estudo foi realizado em creches públicas do município de Campina Grande, Paraíba, pertencentes à Secretaria Municipal de Educação. Ao todo funcionavam, ao momento da coleta de dados, 25 creches em bairros distintos do município, situadas, a maioria, em áreas carentes. Segundo a localização, 23 creches estavam na zona urbana e duas na zona rural. Segundo a faixa etária, oito creches apresentavam atendimento em berçário (crianças entre 4 e 20 meses), 17 atendiam apenas crianças maiores de 20 meses ou mais e $93 \%$ do total de crianças tinham 24 meses ou mais de idade.

O universo de estudo foi de 2.749 crianças devidamente cadastradas e frequentando as creches, assim distribuídas: 2.473 na zona urbana e 276 na zona rural; sendo 199 atendidas em berçário. A população elegível incluiu todas as crianças, exceto as gêmeas, as adotadas, as de mães com idade inferior a 18 anos (condição associada a complicações no estado de saúde e nutrição do filho) e aquelas com problemas físicos que dificultassem a avaliação antropométrica. No caso de crianças irmãs nas creches, uma delas foi sorteada para o estudo.

O cálculo para estimar o tamanho da amostra baseou-se no procedimento para descrição da proporção. Considerou-se uma prevalência estimada (p) de déficit de estatura em crianças menores de cinco anos de $7,0 \%{ }^{13}$, um erro amostral (d) de $3 \%$ e um nível de $95 \%$ de confiança $\left(Z \alpha^{2}=\right.$ $\left.1,96^{2}\right)$, utilizando a fórmula:

$$
\mathrm{n}=\frac{\mathrm{N}^{\star} \mathrm{Z}_{\alpha}^{2 *} p^{\star} q}{\mathrm{~d} 2 *(\mathrm{~N}-1)+\mathrm{Z}_{\alpha}^{2 *} p^{\star} q}
$$

O valor calculado (252) foi acrescido em 10\% para perdas e recusas e efeito de delineamento amostral de 1,2, perfazendo uma amostra de 335 sujeitos. Tamanhos amostrais proporcionais foram considerados para o estudo de crianças segundo a zona de localização da creche (urbana, 
rural) e a idade da criança (menores de dois anos, dois anos ou mais).

Para a seleção da amostra, 14 creches (quantidade representativa do total e suficiente para conformação da amostra) foram selecionadas por sorteio aleatório simples, sendo uma selecionada entre as localizadas na zona rural e duas entre aquelas com atendimento de berçário. Posteriormente, com posse da lista das crianças assistidas nas creches, foram selecionadas de forma sistemática 15 crianças de 24 meses ou mais por creche de pequeno porte ( 3 creches), 20 por creche de mediano porte ( 3 creches), 25 por creche de grande porte ( 5 creches) e 35 na creche sorteada da zona rural. Em cada uma das duas creches sorteadas com atendimento de berçário, foram selecionadas 35 crianças menores de dois anos.

No presente estudo, além de dados antropométricos, foram contempladas informações sobre as condições socioeconômicas (escoamento sanitário; coleta do lixo; presença de sanitário; abastecimento da água; tratamento da água para beber; posse de bens duráveis - TV, DVD, geladeira, máquina de lavar, carro; número de pessoas no domicílio; número de pessoas que dormem juntas no mesmo cômodo que a criança; benefício de programa social), as características maternas (trabalho fora de casa, alfabetização, número de consultas de pré-natal) e o perfil das crianças (idade, sexo, peso ao nascer, percepção materna da saúde, tempo de permanência na creche).

As crianças menores de dois anos tiveram o comprimento medido por meio de antropômetro infantil de madeira (Alturexata ${ }^{\circledR}$ ) com amplitude de $130 \mathrm{~cm}$ e subdivisões de $0,1 \mathrm{~cm}$. As crianças de dois anos ou mais tiveram a altura medida por meio de estadiômetro (WCS ${ }^{\circledR}$ ) com amplitude de $200 \mathrm{~cm}$ e subdivisões de 0,1 $\mathrm{cm}$. Todas as crianças foram pesadas utilizando balança eletrônica do tipo plataforma com capacidade para $150 \mathrm{~kg}$ e graduação em $100 \mathrm{~g}$ (Tanita $\left.\mathrm{UM}-080^{\circledR}\right)$. Na pesagem, foi permitida apenas uma peça intima leve e no caso de crianças que usavam fraldas, estas foram retiradas. $\mathrm{O}$ peso de crianças de colo foi calculado pela diferença entre o peso da mãe com a criança no colo e o peso da mãe. As medições foram realizadas em duplicata, aceitando-se variação máxima de $0,3 \mathrm{~mm}$, e a medida final resultou da estimativa da média das duas medições. As medições foram realizadas de acordo com normas técnicas padronizadas, obedecendo aos procedimentos recomendados pela Organização Mundial da Saúde ${ }^{14}$.

Os escores- $Z$ de peso/estatura e peso/idade foram calculados com o programa WHO Anthro
2009. Tomou-se como referência a população do Multicentre Growth Reference Study, atualmente recomendado pela Organização Mundial da Saú$\mathrm{de}^{15}$.

As informações socioeconômicas, maternas e da percepção das mães sobre a saúde das crianças foram obtidas utilizando-se questionário estruturado aplicado às mães das crianças. A data de nascimento, o sexo e o peso ao nascer das crianças foram retirados da caderneta de saúde. A idade da criança foi calculada em meses, mediante a diferença entre a data de nascimento e a data da entrevista. O tempo de permanência das crianças na creche foi uma informação conhecida na etapa de amostragem. A alfabetização das mães considerou a capacidade de ler, escrever e fazer contas por escrito.

Com o objetivo de assegurar a validação da digitação, os dados foram digitados com dupla entrada, através do programa Excel (Microsoft Inc., Estados Unidos). Após o término da digitação, os dois bancos de dados foram cruzados com a utilização do aplicativo Validate do programa Epi Info v. 6.04b (WHO/CDC, Atlanta, Estados Unidos), possibilitando assim verificar a consistência dos dados e gerando o banco final que foi usado para análise estatística. As análises estatísticas foram realizadas através do programa Rv2.10.0. Para testar a suposição de normalidade das variáveis envolvidas no estudo foi aplicado o teste de Shapiro Wilk.

O peso/estatura e o peso/idade, expressos em escores- $Z$, foram analisados como variáveis contínuas dependentes. O teste t de Student foi utilizado para estabelecer as diferenças entre médias nas análises bivariadas, considerando-se significância estatística um valor $\mathrm{p}<0,05$. Todas as variáveis com valor de $p<0,25$ na análise bivariada foram selecionadas para inclusão inicial na análise de regressão. Previamente à regressão linear múltipla, para analisar a existência de multicolinearidade entre as variáveis independentes, foi construída uma matriz com as mesmas e determinou-se o nível de correlação através do teste de correlação de Spearman na tentativa de excluir variáveis colineares $(r>0,80)$, de modo que a matriz de correlação não identificou multicolinearidade.

A análise de regressão linear múltipla (y $=\beta 0+\beta 1^{\star} \mathrm{x} 1 \ldots . \beta \mathrm{n}^{\star} \mathrm{xn}$, sendo $\beta 0$ o coeficiente médio no período e $\beta 1$ o acréscimo ou decréscimo médio para cada variável em análise) foi realizada utilizando-se o modelo hierarquizado, com a finalidade de avaliar o impacto das variáveis explanatórias sobre os índices peso/estatura 
e peso/idade. A abordagem hierárquica consiste na entrada das variáveis de exposição no modelo segundo uma ordem previamente estabelecida fundamentada nas relações lógicas e teóricas da rede de causalidade.

As variáveis foram agrupadas em três blocos: i) variáveis infantis - nível proximal (idade, sexo, peso ao nascer, percepção materna da saúde, tempo de permanência na creche); ii) variáveis maternas - nível intermediário (trabalho fora de casa, alfabetização, número de consultas de prénatal); variáveis socioeconômicas - nível distal (escoamento sanitário; coleta do lixo; presença de sanitário; abastecimento da água; tratamento da água para beber; posse de bens duráveis - TV, DVD, geladeira, máquina de lavar, carro; número de pessoas no domicílio; número de pessoas que dormem juntas no mesmo cômodo que a criança; benefício de programa social). Todas as variáveis independentes foram dicotomizadas, codificando com o valor zero as categorias que foram tomadas como referência e com o valor um as categorias de risco.

Foram rodados vários modelos multivariados de regressão logística, iniciando-se com as variáveis explicativas pertencentes ao nível distal, no qual eram introduzidas uma a uma (método forward), permanecendo as variáveis estatisticamente associadas ao peso/estatura e peso/idade, ao nível de significância de 10\%. Repetiu-se o mesmo passo para os níveis subsequentes (intermediário e proximal). Ao analisar as variáveis do nível intermediário, permaneceram no modelo as do nível distal, mesmo ocorrendo alteração de significância estatística; e assim o mesmo para o nível proximal. Foi considerado o nível de significância de 5\% para indicar associação.

O projeto foi aprovado pelo Comitê de Ética em Pesquisa da Universidade Estadual da Paraíba. Todas as mães cujas crianças foram avaliadas e as diretoras das creches assinaram o Termo de Consentimento Livre e Esclarecido. Os resultados foram divulgados nas instâncias pertinentes através de encontros com a Secretaria de Educação do Município e contatos pessoais com os pais ou responsáveis pelas crianças. A divulgação compreendeu o diagnóstico do município, por creche e por criança, bem como orientações nutricionais e de saúde adequadas aos problemas detectados.

\section{Resultados}

Do universo de 2.749 crianças, 2.633 foram consideradas elegíveis para o estudo; foram excluídas 60 por serem gêmeas, 38 por serem adotadas, oito por serem de mães menores de 18 anos e 10 por apresentarem problemas físicos que comprometeria a avaliação antropométrica. Em 14 casos, as crianças sorteadas não compareceram à creche ou não estavam acompanhadas pela mãe no dia da coleta de dados, 13 mães recusaram participar da pesquisa e em nove crianças, por resistência das mesmas, foi impossível realizar a avaliação antropométrica.

Como se observa na Tabela 1, 83,95\% das crianças do estudo possuíam 24 meses ou mais, houve maior proporção de crianças do sexo masculino $(54,8 \%)$ e aproximadamente $50 \%$ eram assistidas nas creches em tempo integral. Do total de crianças, $6,6 \%$ delas apresentaram baixo peso ao nascer $(<2500 \mathrm{~g})$ e $62,54 \%$ das mães indicaram a saúde dos seus filhos como muito boa ou boa. Deste conjunto de variáveis infantis, as crianças $<24$ meses e as com adequado peso ao nascer tiveram maiores médias de escores- $Z$ de peso/estatura e peso/idade do que as $\geq 24$ meses e as com baixo peso ao nascer, respectivamente. Em relação às variáveis maternas, observaramse frequências expressivas de mães sem trabalho $(53,51 \%)$ e analfabetas $(39,1 \%)$, bem como incremento, estatisticamente significativo, na média dos escores- $Z$ de peso/idade sempre que a mãe era alfabetizada.

No que diz respeito às variáveis socioeconômicas, a maioria das famílias dispunha de rede de esgoto $(67,89 \%)$, de sanitário no domicílio (79,59\%), água tratada para beber $(81,93 \%)$, rede pública para o abastecimento de água $(84,28 \%)$ e coleta de lixo $(91,3 \%)$. Quanto aos bens de consumo duráveis, TV $(98,3 \%)$ e DVD $(90,6 \%)$ foram os de maior posse, enquanto a máquina de lavar $(50,5 \%)$ e o carro $(30,7 \%)$ foram os de menor posse. Apenas $28,76 \%$ dos domicílios eram coabitados por seis ou mais pessoas, entretanto em aproximadamente $60 \%$ dos casos três ou mais pessoas dormiam juntas no mesmo cômodo da criança. A maioria das famílias $(73,91 \%)$ auferia benefício de programa social.

Verificaram-se menores médias de escores- $Z$ de peso/estatura nas crianças de famílias em domicílios sem rede de esgoto e que não dispunham de geladeira (Tabela 2). Para o índice peso/idade, as médias dos escores- $Z$ foram inferiores nas crianças que viviam em domicílios sem coleta do lixo, sem sanitário, de seis ou mais pessoas, com 
Tabela 1. Características da amostra de pré-escolares segundo variáveis infantis, maternas e socioeconômicas. Campina Grande, PB, 2011.

\begin{tabular}{|c|c|c|c|c|c|}
\hline Variáveis & $\mathbf{n}$ & $\%$ & Variáveis & $\mathbf{n}$ & $\%$ \\
\hline Infantis - Nível Proximal & & & Abastecimento de água & & \\
\hline Idade (meses) & & & Rede pública & 252 & 84,28 \\
\hline$\geq 24$ & 251 & 83,95 & Outros & 47 & 15,72 \\
\hline$<24$ & 48 & 16,05 & Tratamento da água para beber & & \\
\hline Sexo & & & Sim & 245 & 81,93 \\
\hline Masculino & 164 & 54,85 & Não & 54 & 18,06 \\
\hline Feminino & 135 & 45,15 & Presença de sanitário no domicílio & & \\
\hline Peso ao nascer(g) & & & Sim & 230 & 79,59 \\
\hline$\geq 2500$ & 269 & 93,4 & Não & 69 & 23,07 \\
\hline$<2500$ & 19 & 6,6 & TV & & \\
\hline Percepção materna da saúde & & & Sim & 294 & 98,33 \\
\hline Muito boa/boa & 187 & 62,54 & Não & 5 & 1,67 \\
\hline Regular/ruim & 112 & 37,46 & DVD & & \\
\hline Tempo de permanência na creche & & & Sim & 271 & 90,64 \\
\hline Integral & 148 & 49,50 & Não & 28 & 9,36 \\
\hline Parcial & 151 & 50,50 & Geladeira & & \\
\hline Maternas - Nível Intermediário & & & Sim & 263 & 87,96 \\
\hline Trabalho fora de casa & & & Não & 36 & 12,04 \\
\hline Não & 160 & 53,51 & Máquina de lavar & & \\
\hline Sim & 139 & 46,49 & Sim & 151 & 50,5 \\
\hline Alfabetizada & & & Não & 148 & 49,5 \\
\hline Sim & 182 & 60,87 & Carro & & \\
\hline Não & 117 & 39,13 & Sim & 92 & 30,77 \\
\hline No de consultas de pré-natal & & & Não & 207 & 69,33 \\
\hline$\geq 6$ & 239 & 82,41 & No de pessoas no domicílio & & \\
\hline$<6$ & 51 & 17,59 & $<6$ & 213 & 71,23 \\
\hline Socioeconômicas - Nível Distal & & & $\geq 6$ & 86 & 28,76 \\
\hline Escoamento sanitário & & & No de pessoas que dormem juntas & & \\
\hline Rede de esgoto & 203 & 67,89 & no mesmo cômodo que a criança & & \\
\hline Outros & 96 & 32,11 & $<3$ & 123 & 41,13 \\
\hline Coleta do lixo & & & $\geq 3$ & 176 & 58,86 \\
\hline Sim & 273 & 91,30 & Benefício de programa social & & \\
\hline \multirow[t]{2}{*}{ Não } & 26 & 8,7 & Sim & 221 & 73,91 \\
\hline & & & Não & 78 & 26,08 \\
\hline
\end{tabular}

três ou mais pessoas dormindo juntas no cômodo da criança, sem geladeira e com benefício de programa social.

As análises dos efeitos combinados das variáveis explanatórias em relação aos índices peso/estatura e peso/idade, condicionado aos resultados das respectivas análises bivariadas (Tabelas 2 e 3), encontram-se nas tabelas 4 e 5 , respectivamente. Para o índice peso/estatura, o modelo 1 revelou que a ausência de geladeira influenciou significativamente os resultados deste índice, de forma negativa. $\mathrm{O}$ modelo 2 mostra que a ausência de rede de esgoto e o uso de água não tratada para beber alcançaram significância estatística, enquanto o trabalho materno e o número de con- sultas de pré-natal não foram estatisticamente significantes após ajuste para as variáveis distais (socioeconômicas). Por fim, a entrada das variáveis proximais, no modelo 3 , revelou, após ajuste para as demais variáveis, perdas nos escores- $Z$ de peso/estatura das crianças quando as mesmas residiam em domicílios sem geladeira, conviviam em condições de água para beber não tratada e nasceram com baixo peso (Tabela 4). Para o caso do índice peso/idade, as condições socioeconômicas (ausência de geladeira, número de pessoas dormindo com a criança $\geq 3$, não coleta do lixo) e o baixo peso ao nascer foram os determinantes que influenciaram negativamente os escores- $Z$ (Tabela 5). 
Tabela 2. Índice peso/estatura, expresso em escore-Z, de pré-escolares, segundo variáveis infantis, maternas e socioeconômicas. Campina Grande, PB, 2011.

\begin{tabular}{|c|c|c|c|c|}
\hline \multirow[b]{2}{*}{ Variáveis } & \multicolumn{3}{|c|}{ Peso/Estatura } & \multirow[b]{2}{*}{ p-valor } \\
\hline & Média & DP & IC95\% & \\
\hline \multicolumn{5}{|l|}{ Infantis - Nível Proximal } \\
\hline Idade (meses) & 0,45 & 1,05 & $-2,72-3,84$ & \multirow[t]{2}{*}{0,0067} \\
\hline $\begin{array}{l}\geq 24 \\
<24\end{array}$ & 1,20 & 1,02 & $-0,53-2,84$ & \\
\hline \multicolumn{5}{|l|}{$\begin{array}{l}<24 \\
\text { Sexo }\end{array}$} \\
\hline Masculino & 0,47 & 1,10 & $-2,72-3,84$ & \multirow[t]{2}{*}{0,3490} \\
\hline Feminino & 0,51 & 1,01 & $-1,63-3,77$ & \\
\hline Peso ao nascer $(\mathrm{g})$ & 0,55 & 1,05 & $-2,72-3,84$ & \multirow[t]{2}{*}{0,0014} \\
\hline $\begin{array}{l}\geq 2500 \\
<2500\end{array}$ & $-0,26$ & 1,02 & $-2,18-2,06$ & \\
\hline \multicolumn{5}{|l|}{ Percepção materna da saúde } \\
\hline Muito boa/boa & 0,42 & 1,01 & $-2,72-3,84$ & \multirow{2}{*}{0,8983} \\
\hline Regular/ruim & 0,42 & & & \\
\hline Tempo de permanência na creche & 0,59 & 1,03 & $-1,58-3,77$ & \multirow[t]{2}{*}{0,1014} \\
\hline $\begin{array}{l}\text { Integral } \\
\text { Parcial }\end{array}$ & 0,39 & 1,09 & $-2,72-3,84$ & \\
\hline \multicolumn{5}{|l|}{ Maternas - Nível Intermediário } \\
\hline Trabalho fora de casa & & & & \multirow{3}{*}{0,1266} \\
\hline Não & 0,40 & $\begin{array}{l}1,00 \\
1,06\end{array}$ & $-2,12-5,04$ & \\
\hline $\operatorname{Sim}$ & 0,59 & 1,06 & $-1,63-3,77$ & \\
\hline \multicolumn{3}{|l|}{ Alfabetizada } & $-2,18-3,75$ & \multirow[t]{3}{*}{0,9143} \\
\hline Sim & 0,50 & 1,11 & $-2,72-3,84$ & \\
\hline Não & & & & \\
\hline No de consultas de pré-natal & 0,59 & 1,13 & $-1,57-2,92$ & \multirow{3}{*}{0,1738} \\
\hline$\geq 6$ & 0,44 & 1,04 & $-2,72-3,84$ & \\
\hline$<6$ & & & & \\
\hline
\end{tabular}

continua

\section{Discussão}

De modo semelhante ao evidenciado no presente estudo, postula-se que o estado nutricional das crianças associa-se a fatores de diferentes níveis de complexidade, a exemplo das condições socioeconômicas da família, das características maternas e das características da própria criança ${ }^{16}$. O conhecimento desses fatores é essencial para o planejamento e a monitoração de programas e ações relacionados à melhoria do estado nutricional $^{17}$.

Apesar de que neste estudo um maior tempo de permanência na creche não representou diferenças nos índices peso/estatura e peso/idade, resultados sistematizados da literatura sugerem a melhoria do estado nutricional condicionado pela frequência ${ }^{18,19}$. O confronto entre esses resultados pode ser explicado considerando a complexidade do estado nutricional, com representações próprias para cada tempo e espaço ${ }^{20}$. Por outro lado, talvez, a relação do estado nutricional com a frequência à creche seja mais convincente para a estatura/idade, do que para o peso/estatura e o peso/idade, assemelhando-se, segundo resultados de âmbito nacional, às maiores perdas na estatura/idade, do que nos outros índices antropométricos, decorrentes de situações de insegurança alimentar ${ }^{21}$. Nesse sentido, há que enfatizar o papel das creches como espaço privilegiado de promoção da segurança alimentar e nutricional ${ }^{18}$ e na oferta de cuidados integrais que protegem o potencial de crescimento ${ }^{22}$.

A influência do peso ao nascer no estado nutricional de crianças, observada por outros pesquisadores ${ }^{8,23}$, é sugerida, também, pelos dados desta pesquisa, ao constatar, no modelo ajustado, a sua relevância entre todos os fatores considerados no bloco de variáveis infantis, tanto para o peso/idade quanto para o peso/estatura. Em 
Tabela 2. continuação

\begin{tabular}{|c|c|c|c|c|}
\hline \multirow[b]{2}{*}{ Variáveis } & \multicolumn{3}{|c|}{ Peso/Estatura } & \multirow[b]{2}{*}{ p-valor } \\
\hline & Média & DP & IC95\% & \\
\hline \multicolumn{5}{|c|}{ Socioeconômicas - Nível Distal } \\
\hline \multicolumn{5}{|l|}{ Escoamento sanitário } \\
\hline Rede de esgoto & 0,67 & 1,09 & $-2,72-3,84$ & \multirow[t]{2}{*}{0,0329} \\
\hline Outros & 0,40 & 1,04 & $-2,18-3,77$ & \\
\hline \multicolumn{5}{|l|}{ Coleta do lixo } \\
\hline Sim & 0,50 & 1,09 & $-2,72-3,84$ & \multirow[t]{2}{*}{0,8179} \\
\hline Não & 0,41 & 0,68 & $-0,67-1,83$ & \\
\hline \multicolumn{5}{|l|}{ Abastecimento de água } \\
\hline Rede pública & 0,50 & 1,06 & $-2,72-3,84$ & \multirow[t]{2}{*}{0,7470} \\
\hline Outros & 0,44 & 1,07 & $-1,46-2,92$ & \\
\hline \multicolumn{5}{|c|}{ Tratamento da água para beber } \\
\hline Sim & 0,53 & 1,09 & $-2,72-3,84$ & \multirow[t]{2}{*}{0,0935} \\
\hline Não & 0,30 & 0,9 & $-1,41-3,05$ & \\
\hline \multicolumn{5}{|c|}{ Presença de sanitário no domicílio } \\
\hline Sim & 0,53 & 1,12 & $-2,72-3,84$ & \multirow[t]{2}{*}{0,2747} \\
\hline Não & 0,35 & 0,82 & $-1,41-2,09$ & \\
\hline \multicolumn{5}{|l|}{ TV } \\
\hline Sim & 0,50 & 1,06 & $-2,72-3,84$ & \multirow[t]{2}{*}{0,2106} \\
\hline Não & 0,03 & 0,97 & $-0,83-1,66$ & \\
\hline \multicolumn{5}{|l|}{ DVD } \\
\hline Sim & 0,50 & 1,07 & $-2,72-3,84$ & \multirow{2}{*}{0,9963} \\
\hline Não & 0,41 & 0,94 & $-1,63-1,87$ & \\
\hline \multicolumn{5}{|l|}{ Geladeira } \\
\hline Sim & 0,55 & 1,06 & $-2,72-3,84$ & \multirow[t]{2}{*}{0,0091} \\
\hline Não & 0,04 & 0,97 & $-1,63-1,91$ & \\
\hline \multicolumn{5}{|l|}{ Máquina de lavar } \\
\hline Sim & 0,45 & 1,07 & $-2,72-3,77$ & \multirow[t]{2}{*}{0,5674} \\
\hline Não & 0,53 & 1,06 & $-2,18-3,84$ & \\
\hline \multicolumn{5}{|l|}{ Carro } \\
\hline Sim & 0,44 & 1,10 & $-2,72-3,84$ & \multirow[t]{2}{*}{0,7416} \\
\hline Não & 0,51 & 1,04 & $-2,18-3,77$ & \\
\hline \multicolumn{5}{|c|}{ No de pessoas no domicílio } \\
\hline$<6$ & 0,53 & 1,07 & $-2,72-3,84$ & 0,2989 \\
\hline$\geq 6$ & 0,39 & 1,04 & $-1,63-3,77$ & \\
\hline No de pessoas que dorr & & & & \\
\hline no mesmo cômodo qu & & & & \\
\hline$<3$ & 0,5 & 1,1 & $-2,18-3,84$ & 0,6884 \\
\hline$\geq 3$ & 0,48 & 1,04 & $-2,72-3,77$ & \\
\hline Benefício de programa & & & & \\
\hline Sim & 0,45 & 1,05 & $-2,72-3,77$ & 0,3769 \\
\hline Não & 0,60 & 1,05 & $-1,52-3,84$ & \\
\hline
\end{tabular}

p-valor: t-Student.

estudo tipo caso-controle aninhado a uma coorte na zona da Mata Meridional de Pernambuco, cujo objetivo principal foi analisar a associação entre o baixo peso ao nascer e o estado nutricional ao final do primeiro ano de vida, os pesquisadores apontaram o baixo peso ao nascer como um dos principais fatores responsáveis pelo risco nutricional avaliado por meio do índice peso/ idade. Os autores argumentam que as crianças que nascem com baixo peso, independente do crescimento compensatório nos três primeiros meses de vida, permanecem abaixo das crianças com peso de nascimento adequado, contribuindo para uma maior vulnerabilidade a processos 
Tabela 3. Índice peso/idade, expressos em escore-Z, de pré-escolares, segundo variáveis infantis, maternas e socioeconômicas. Campina Grande, PB, 2011.

\begin{tabular}{|c|c|c|c|c|}
\hline \multirow[b]{2}{*}{ Variáveis } & \multicolumn{3}{|c|}{ Peso/Idade } & \multirow[b]{2}{*}{ p-valor } \\
\hline & Média & DP & IC95\% & \\
\hline \multirow{2}{*}{\multicolumn{5}{|c|}{$\begin{array}{l}\text { Infantis - Nível Proximal } \\
\text { Idade (meses) }\end{array}$}} \\
\hline & & & & \\
\hline$\geq 24$ & 0,06 & 1,08 & $-3,27-3,27$ & \multirow[t]{2}{*}{0,0332} \\
\hline$<24$ & 0,66 & 0,99 & $-1,27-2,20$ & \\
\hline \multicolumn{5}{|l|}{ Sexo } \\
\hline Masculino & 0,08 & 1,11 & $-3,09-3,13$ & \multirow[t]{2}{*}{0,8695} \\
\hline Feminino & 0,10 & 1,05 & $-3,27-3,27$ & \\
\hline \multicolumn{5}{|l|}{ Peso ao nascer(g) } \\
\hline$\geq 2500$ & 0,15 & 1,07 & $-3,27-3,27$ & \multirow[t]{2}{*}{0,0005} \\
\hline$<2500$ & $-0,75$ & 1,05 & $-2,29-1,33$ & \\
\hline \multicolumn{5}{|c|}{ Percepção materna da saúde } \\
\hline Muito boa/boa & 0,16 & 1,10 & $-3,27-3,27$ & \multirow[t]{2}{*}{0,3055} \\
\hline Regular/ruim & $-0,03$ & 1,04 & $-3,09-3,13$ & \\
\hline \multicolumn{5}{|c|}{ Tempo de permanência na creche } \\
\hline Integral & 0,02 & 1,10 & $-3,27-3,27$ & \multirow[t]{2}{*}{0,3459} \\
\hline Parcial & 0,15 & 1,06 & $-3,09-3,13$ & \\
\hline \multicolumn{5}{|c|}{ Maternas - Nível Intermediário } \\
\hline \multicolumn{5}{|l|}{ Trabalho fora de casa } \\
\hline Não & $-0,02$ & 1,11 & $-3,09-3,13$ & \multirow[t]{2}{*}{0,0743} \\
\hline Sim & 0,21 & 1,03 & $-3,27-3,27$ & \\
\hline \multicolumn{5}{|l|}{ Alfabetizada } \\
\hline Sim & 0,18 & 0,98 & $-2,21-2,53$ & \multirow[t]{2}{*}{0,0425} \\
\hline Não & $-0,06$ & 1,21 & $-3,27-3,27$ & \\
\hline \multicolumn{5}{|c|}{ No de consultas de pré-natal } \\
\hline$\geq 6$ & 0,15 & 1,18 & $-2,29-3,06$ & \multirow[t]{2}{*}{0,6496} \\
\hline$<6$ & 0,06 & 1,07 & $-3,27-3,27$ & \\
\hline
\end{tabular}

continua

infecciosos e outros fatores pós-natais negativos, os quais agregam-se à programação de crescimento pré-natal ${ }^{24}$.

Em relação ao peso/estatura, sugere-se que o baixo peso ao nascer pode resultar em mecanismos de adaptação, como o catch-upgrowth e distúrbios hormonais, que poderiam predispor ao desenvolvimento de sobrepeso/obesidade ${ }^{25}$. Nesse sentido, a promoção do adequado peso ao nascer deve tratar as questões relacionadas ao estado nutricional pré-gestacional e à alimentação durante a gravidez ${ }^{26}$. Essa conjuntura descrita apresenta grande relevância atual, uma vez que se sugerem o sobrepeso/obesidade como condições nutricionais de importante magnitude epidemiológica entre as crianças brasileiras, inclusive as institucionalizadas em creches, sobretudo nas regiões Sul e Nordeste, com aumento progressivo das $\operatorname{taxas}^{27-29}$.
As características maternas, representadas pela escolaridade, trabalho fora de casa, entre outras, são apontadas como importantes preditores do estado nutricional infantil, à medida que influenciam diretamente na prestação de cuidados apropriados para a promoção da saúde e nutrição da criança ${ }^{16,30}$. Por outro lado, em relação ao peso/estatura, distingue-se uma distribuição equitativa do sobrepeso/obesidade em países em desenvolvimento ${ }^{31}$, inclusive no Brasil ${ }^{28}$. Neste estudo, nenhuma das características maternas, quando ajustadas para as outras variáveis, mostrou-se fator potencial com influência no estado nutricional das crianças investigadas. Esse fato, além de poder expressar a contextualização mais marcante da situação socioeconômica, em relação ao perfil materno, sobretudo para o índice peso/idade, pode indicar a necessidade de análises longitudinais para maior clareza. Com rela- 
Tabela 2. continuação

\begin{tabular}{|c|c|c|c|c|}
\hline \multirow[b]{2}{*}{ Variáveis } & \multicolumn{3}{|c|}{ Peso/Idade } & \multirow[b]{2}{*}{ p-valor } \\
\hline & Média & DP & IC95\% & \\
\hline \multicolumn{5}{|l|}{ Socioeconômicas - Nível Distal } \\
\hline \multicolumn{5}{|l|}{ Escoamento sanitário } \\
\hline Rede de esgoto & 0,22 & 1,13 & $-3,09-3,13$ & \multirow{2}{*}{0,1487} \\
\hline Outros & 0,02 & 1,05 & $-3,27-3,27$ & \\
\hline \multicolumn{5}{|l|}{ Coleta do lixo } \\
\hline Sim & 0,12 & 1,09 & $-3,27-3,27$ & \multirow[t]{2}{*}{0,0399} \\
\hline Não & $-0,31$ & 0,85 & $-2,09-1,41$ & \\
\hline \multicolumn{5}{|l|}{ Abastecimento de água } \\
\hline Rede pública & 0,10 & 1,07 & $-3,27-3,27$ & \multirow[t]{2}{*}{0,5290} \\
\hline Outros & $-0,01$ & 1,14 & $-2,27-3,06$ & \\
\hline \multicolumn{5}{|l|}{ Tratamento da água para beber } \\
\hline Sim & 0,14 & 1,09 & $-3,27-3,27$ & \multirow[t]{2}{*}{0,0715} \\
\hline Não & $-0,15$ & 1,03 & $-2,27-3,01$ & \\
\hline \multicolumn{5}{|l|}{ Presença de sanitário no domicílio } \\
\hline Sim & 0,18 & 1,13 & $-3,27-3,27$ & \multirow[t]{2}{*}{0,0027} \\
\hline Não & $-0,21$ & 0,86 & $-2,29-1,27$ & \\
\hline \multicolumn{5}{|l|}{ TV } \\
\hline Sim & 0,10 & 1,07 & $-3,27-3,27$ & \multirow[t]{2}{*}{0,1170} \\
\hline Não & $-0,73$ & 1,29 & $-2,09-1,12$ & \\
\hline \multicolumn{5}{|l|}{ DVD } \\
\hline Sim & 0,12 & 1,08 & $-3,27-3,27$ & \multirow[t]{2}{*}{0,2689} \\
\hline Não & $-0,21$ & 1,11 & $-2,52-1,67$ & \\
\hline \multicolumn{5}{|l|}{ Geladeira } \\
\hline Sim & 0,16 & 1,07 & $-3,27-3,27$ & \multirow[t]{2}{*}{0,0030} \\
\hline Não & $-0,47$ & 0,97 & $-2,52-1,12$ & \\
\hline \multicolumn{5}{|l|}{ Máquina de lavar } \\
\hline Sim & 0,14 & 1,09 & $-3,27-3,27$ & \multirow[t]{2}{*}{0,4085} \\
\hline Não & 0,03 & 1,07 & $-2,52-3,13$ & \\
\hline \multicolumn{5}{|l|}{ Carro } \\
\hline Sim & 0,17 & 1,14 & $-3,27-3,13$ & \multirow[t]{2}{*}{0,3961} \\
\hline Não & 0,05 & 1,06 & $-2,52-3,27$ & \\
\hline \multicolumn{5}{|l|}{ No de pessoas no domicílio } \\
\hline$<6$ & 0,17 & 1,05 & $-3,09-3,13$ & 0,0379 \\
\hline$\geq 6$ & $-0,13$ & 1,14 & $-3,27-3,27$ & \\
\hline No de pessoas que dormem juntas & & & & \\
\hline no mesmo cômodo que a criança & & & & 0,0106 \\
\hline$<3$ & 0,3 & 0,99 & $-1,95-3,13$ & \\
\hline$\geq 3$ & $-0,06$ & 1,12 & $-3,27-3,27$ & \\
\hline Benefício de programa social & & & & 0,0241 \\
\hline Sim & 0 & 1,08 & $-3,27-3,27$ & \\
\hline Não & 0,32 & 1,06 & $-2,29-3,13$ & \\
\hline
\end{tabular}

p-valor: t-Student.

ção ao peso/estatura, os resultados obtidos podem refletir a tendência relacionada à paridade no risco de desvios em díspares contextos.

Nas crianças estudadas, o fator de variação mais importante no estado nutricional esteve no conjunto das variáveis socioeconômicas, principalmente quando analisado o peso/idade. A determinação socioeconômica do estado nutricio- nal antropométrico de pré-escolares brasileiros tem sido constatada por estudos transversais ${ }^{7,32}$ e longitudinais ${ }^{33,34}$ que trabalharam com os mesmos índices. Constatações similares também têm sido obtidas em localidades de outros países em desenvolvimento ${ }^{35-37}$. É possível interpretar esses resultados como indicativos de que a melhoria nas condiçõoes de vida das crianças é crucial para 
Tabela 4. Modelo de regressão linear hierarquizado de fatores determinantes do índice peso/estatura de crianças pré-escolares. Campina Grande, PB, 2011.

\begin{tabular}{|c|c|c|c|c|c|c|}
\hline \multirow{2}{*}{ Variáveis } & \multicolumn{2}{|c|}{$\begin{array}{c}\text { Modelo } 1 \\
\text { Distal }\end{array}$} & \multicolumn{2}{|c|}{$\begin{array}{c}\text { Modelo 2 } \\
\text { Distal+Intermediário } \\
\end{array}$} & \multicolumn{2}{|c|}{$\begin{array}{c}\text { Modelo } 3 \\
\text { Distal+Intermediário+Proximal } \\
\end{array}$} \\
\hline & $\beta$ & p-valor & $\beta$ & p-valor & $\beta$ & p-valor \\
\hline \multicolumn{7}{|l|}{ Socioeconômicas } \\
\hline \multicolumn{7}{|l|}{ Geladeira } \\
\hline Não & $-0,51$ & 0,0056 & $-0,52$ & 0,0053 & $-0,54$ & 0,0037 \\
\hline \multicolumn{7}{|l|}{ Escoamento sanitário } \\
\hline Diferente de rede de esgoto & 0,24 & 0,0720 & 0,27 & 0,0439 & 0,21 & 0,1182 \\
\hline \multicolumn{7}{|l|}{ Tratamento da água para beber } \\
\hline Não & $-0,28$ & 0,0710 & $-0,36$ & 0,0264 & $-0,33$ & 0,0486 \\
\hline \multicolumn{7}{|l|}{ Maternas } \\
\hline \multicolumn{7}{|l|}{ Trabalho fora de casa } \\
\hline Não & & & 0,16 & 0,1922 & 0,07 & 0,5934 \\
\hline \multicolumn{7}{|l|}{ No de consultas de pré-natal } \\
\hline$<6$ & & & 0,21 & 0,1934 & 0,23 & 0,1528 \\
\hline \multicolumn{7}{|l|}{ Infantis } \\
\hline \multicolumn{7}{|l|}{ Peso ao nascer(g) } \\
\hline$<2500$ & & & & & $-0,73$ & 0,0030 \\
\hline \multicolumn{7}{|l|}{ Idade(meses) } \\
\hline$<24$ & & & & & 0,45 & 0,1280 \\
\hline \multicolumn{7}{|l|}{ Tempo de permanência na creche } \\
\hline Parcial & & & & & $-0,19$ & 0,1356 \\
\hline
\end{tabular}

$\beta$ = Coeficiente de regressão; Modelo 1: Variáveis ajustadas umas pelas outras; Modelo 2: Ajustado pelas variáveis do Modelo 1; Modelo 3: Ajustado pelas variáveis dos Modelos 1 e 2; Resultado com significância em negrito. Coeficiente de determinação $\left(\mathrm{R}^{2}\right)$ : $16,4 \%$.

que elas possam expressar seu pleno potencial de crescimento e desenvolvimento, como observado por outros autores ${ }^{17}$. Assim, esses resultados apontam a importância que a situação socioeconômica desfavorável continua exercendo no estado nutricional, apesar do processo de transição epidemiológica e nutricional experimentado no país. Destarte, sugerem-se, ainda, desafios imediatos relacionados à erradicação da fome e pobreza extrema como parte dos objetivos para 2015 estabelecidos nos Objetivos de Desenvolvimento do Milênio, cuja medição deve ser realizada com a utilização do índice peso/idade.

Cabe frisar que outros estudos transversais desenvolvidos em diferentes localidades do país, ao analisar a influência das condições socioeconômicas nos índices peso/estatura e peso/idade, apresentam-se com resultados dissímeis. A associação com as condições sanitárias inadequadas, por exemplo, foi encontrada em algumas localidades do país ${ }^{38,39}$ e em outras não ${ }^{4,40,41}$. Situação similar para o benefício do Programa Bolsa Família, que foi associado a maior vulnerabilidade nutricional segundo o peso/idade em crianças de Paula Cândido, $\mathrm{MG}^{38}$, contrariamente ao verifi- cado em João Câmara, $\mathrm{RN}^{42}$ e no presente estudo. Nesse sentido, há que ressaltar a importância do desenvolvimento de pesquisas de desenho longitudinal no estudo de processos dinâmicos como o crescimento e desenvolvimento ${ }^{18}$ e, por sua vez, as limitações dos estudos transversais no estabelecimento de relação causal ${ }^{40}$. Ainda, mesmo que os resultados possam diferir, a importância desses fatores no estado nutricional decorre, também, por suas implicações na condição de saúde. A posse de geladeira, por exemplo, é importante na prevenção da contaminação de alimentos e, portanto, na redução de doenças diarreicas. Assim, também, a presença de saneamento no domicílio constitui um indicador indireto de higiene e de cuidados associados à ocorrência de infecções de repetição ${ }^{24}$. $\mathrm{O}$ acesso à infraestrutura de saneamento ambiental constitui um dos fatores associados às melhorias nos índices de desnutrição e, portanto, deve ser priorizado como medida preventiva ${ }^{43}$.

Alguns fatores relacionados ao estado nutricional das crianças não foram considerados neste estudo, como a renda familiar, a segurança alimentar e nutricional das famílias, e as práticas 
Tabela 5. Modelo de regressão linear hierarquizado de fatores determinantes do índice peso/idade de crianças pré-escolares. Campina Grande, PB, 2011.

\begin{tabular}{|c|c|c|c|c|c|c|}
\hline \multirow{2}{*}{ Variáveis } & \multicolumn{2}{|c|}{$\begin{array}{c}\text { Modelo } 1 \\
\text { Distal }\end{array}$} & \multicolumn{2}{|c|}{$\begin{array}{c}\text { Modelo 2 } \\
\text { Distal+Intermediário } \\
\end{array}$} & \multicolumn{2}{|c|}{$\begin{array}{c}\text { Modelo } 3 \\
\text { Distal+Intermediário+Proximal } \\
\end{array}$} \\
\hline & $\beta$ & p-valor & $\beta$ & p-valor & $\beta$ & p-valor \\
\hline \multicolumn{7}{|l|}{ Socioeconômicas } \\
\hline \multicolumn{7}{|l|}{ Geladeira } \\
\hline Não & $-0,51$ & 0,0017 & $-0,54$ & 0,0037 & $-0,54$ & 0,0036 \\
\hline \multicolumn{7}{|c|}{$\begin{array}{l}\text { No de pessoas que dormem junto } \\
\text { no mesmo cômodo que à criança }\end{array}$} \\
\hline$\geq 3$ & 0,40 & 0,0286 & $-0,25$ & 0,0480 & $-0,29$ & 0,0232 \\
\hline \multicolumn{7}{|l|}{ Benefício de programa social } \\
\hline $\operatorname{Sim}$ & $-0,23$ & 0,0581 & 0,23 & 0,0940 & 0,23 & 0,1059 \\
\hline \multicolumn{7}{|l|}{ Coleta do lixo } \\
\hline Não & $-0,72$ & 0,0073 & $-0,55$ & 0,0153 & $-0,47$ & 0,0385 \\
\hline \multicolumn{7}{|l|}{ Escoamento sanitário } \\
\hline Diferente de rede de esgoto & 0,54 & 0,0476 & 0,33 & 0,0193 & 0,27 & 0,0590 \\
\hline \multicolumn{7}{|l|}{ Maternas } \\
\hline \multicolumn{7}{|l|}{ Alfabetizada } \\
\hline Não & & & $-0,17$ & 0,2039 & $-0,19$ & 0,1589 \\
\hline \multicolumn{7}{|l|}{ Trabalho fora de casa } \\
\hline Não & & & 0,16 & 0,1836 & 0,10 & 0,4044 \\
\hline \multicolumn{7}{|l|}{ Infantis } \\
\hline \multicolumn{7}{|l|}{ Peso ao nascer(g) } \\
\hline$<2500$ & & & & & $-0,77$ & 0,0018 \\
\hline \multicolumn{7}{|l|}{ Idade(meses) } \\
\hline$<25$ & & & & & 0,39 & 0,1775 \\
\hline
\end{tabular}

$\beta=$ Coeficiente de regressão; Modelo 1: Variáveis ajustadas umas pelas outras; Modelo 2: Ajustado pelas variáveis do Modelo 1; Modelo 3: Ajustado pelas variáveis dos Modelos 1 e 2; Resultado com significância em negrito. Coeficiente de determinação $\left(\mathrm{R}^{2}\right)$ : $13,8 \%$.

alimentares e de atividade física. Nesse sentido, há que destacar as evidências da literatura sobre a situação das crianças brasileiras: i) o aumento do poder aquisitivo como um dos fatores mais importantes relacionados aos ganhos no estado nutricional, inclusive em relação ao peso/ida$\mathrm{de}^{44}$; ii) a relação sistematizada entre a segurança alimentar e o índice peso/idade ${ }^{45}$, bem como a constatação, baseada em dados nacionais, de piores índices nutricionais entre as crianças que sofrem com algum nível de insegurança alimen$\operatorname{tar}^{22}$; e iii) o ambiente propício para o desenvolvimento de sobrepeso/obesidade gerado em condições de desmame precoce, alimentação pouco balanceada e inatividade física ${ }^{46}$.

\section{Conclusões}

Infere-se a tendência do peso ao nascer como fator relevante no peso/estatura e peso/idade das crianças, bem como das condições socioeconômicas, marcadamente no peso/idade. Sugere-se, ainda, que os resultados encontrados expressam circunstâncias nas quais o peso/estatura das crianças comporta-se de maneira mais equitativa. Em relação às condições socioeconômicas, houve indicativo de que não ter geladeira em casa representa um fator de risco para o potencial de crescimento relacionado a ambos os índices. De forma similar, o não tratamento da água de beber, para o crescimento ponderal; e a aglomeração, residir na zona urbana e não dispor de coleta de lixo domiciliar, para o crescimento global. Tais evidências reforçam a importância de considerar a prevenção do baixo peso ao nascer como medida básica direcionada à adequação do estado nutricional, assim como a melhoria das condições socioeconômicas como estratégia que vise a diminuir os desvios de peso/idade que ainda persistem em grupos vulneráveis e que prejudicam o desenvolvimento social. 


\section{Colaboradores}

D Figueroa Pedraza participou da elaboração do projeto, concepção do artigo, revisão bibliográfica, análise e interpretação dos dados, redação e aprovação do artigo. MM Oliveira participou da revisão bibliográfica, análise e interpretação dos dados, redação e aprovação do artigo. MVLML Cardoso participou da revisão crítica e aprovação da versão final. ACD Rocha e EMN Araújo participaram da interpretação dos dados, redação e aprovação do artigo.

\section{Referências}

1. Araújo ACT, Campos JADB. Subsídios para a avaliação do estado nutricional de crianças e adolescentes por meio de indicadores antropométricos. Aliment Nutr 2008; 19(2):219-225.

2. Pereira AS, Vieira CBL, Barbosa RMS, Soares EA, Lanzillotti HS. Análise comparativa do estado nutricional de pré-escolares. Rev Paul Pediatr 2010; 28(2):176-180.

3. Brock RS, Falcão MC. Avaliação nutricional do recémnascido: limitações dos métodos atuais e novas perspectivas. Rev Paul Pediatr 2008; 26(1):70-76.

4. Pinho CPS, Silva JEM, Silva ACG, Araújo NNA, Fernandes CE, Pinto FCL. Avaliação antropométrica de crianças em creches do município de Bezerros, PE. Rev Paul Pediatr 2010; 28(3):315-321.

5. Oliveira JS, Lira PIC, Andrade SLLS, Sales AC, Maia SR, Filho MB. Insegurança Alimentar e estado nutricional de crianças de São João do Tigre, no semi-árido do Nordeste. Rev Bras Epidemiol 2009; 12(3):413-423.

6. Martinez R, Fernandez A. El costo del hambre. Análisis del impacto social y económico de la desnutrición en América Latina: Centroamérica y República Dominicana. Santiago de Chile: CEPAL - PMA; 2007.

7. Menezes RCE, Lira PIC, Oliveira JS, Leal VS, Santana SCS, Andrade SLLS, Filho MB. Prevalência e determinantes do excesso de peso em pré-escolares. $J$ Pediatr 2011; 87(3):231-237.

8. Jesus GM, Vieira GO, Tatiana O Vieira,Martins CC, Mendes CMC, Castelão ES. Fatores determinantes do sobrepeso em crianças menores de 4 anos de idade. $J$ Pediatr 2010; 86(4):311-316.

9. Figueroa Pedraza D. Insegurança alimentar e mortalidade infantil na América Latina: magnitude, características e estratégias de redução com vistas aos objetivos de desenvolvimento do milênio. Saúde Coletiva 2009; 32(6):184-194.

10. Simon VGN, Souza JMP, Leone C, Souza SB. Prevalência de sobrepeso e obesidade em crianças de dois a seis anos matriculadas em escolas particulares no município de São Paulo. Rev Bras Crescimento Desenvolv Hum 2009; 19(2):211-218.

11. Lopes PCS, Prado SRLA, Colombo P. Fatores de risco associados à obesidade e sobrepeso em crianças em idade escolar. Rev Bras Enferm 2010; 63(1):73-78.

12. Bueno MB, Fisberg RM. Comparação de três critérios de classificação de sobrepeso e obesidade entre pré-escolares. Rev Bras Saúde Matern Infant 2006; 6(4):411417.

13. Brasil. Ministério da Saúde (MS). Pesquisa nacional sobre demografia e saúde da criança e da mulher. Brasília: MS; 2008.

14. World Health Organization (WHO). Physical status: the use and interpretation of anthropometry. Geneva: WHO; 1995. (WHO - Technical Report Series, 854).

15. World Health Organization (WHO). WHO Child Growth Standards. Length/height-for-age, weight-forage, weight-for-length, weight-for-height and body mass index-for-age. Methods and development. Geneva: WHO; 2006. 
16. Souza MM, Figueroa Pedraza D, Menezes TN. Estado nutricional de crianças assistidas em creches e situação de (in)segurança alimentar de suas famílias. Cien Saude Colet 2012; 17(12):3425-3436.

17. Oliveira LPM, Barreto ML, Assis AMO, Braga-Junior ATCR, Nunes MFFP, Oliveira NF, Benício MHD, Venâncio SI, Saldiva SRDM, Escuder MML. Preditores do retardo de crescimento linear em pré-escolares: uma abordagem multinível. Cad Saude Publica 2007; 23(3):601-613.

18. Figueroa Pedraza D, Souza MM, Rocha ACD. Fatores associados ao estado nutricional de crianças pré-escolares brasileiras assistidas em creches públicas: uma revisão sistemática. Rev Nutr 2015; 28(4):451-463.

19. Pereira AS, Lanzillotti HS, Soares EA. Frequência à creche e estado nutricional de pré-escolares: uma revisão sistemática. Rev Paul Pediatr 2010; 28(4):366-372.

20. Figueroa Pedraza D. Preditores de riscos nutricionais de crianças assistidas em creches em município de porte médio do Brasil. Cad Saude Colet 2016; no prelo.

21. Santos LPD, Gigante DP. Relação entre insegurança alimentar e estado nutricional de crianças brasileiras menores de cinco anos. Rev Bras Epidemiol 2013; 16(4):984-994.

22. Oliveira JS, Lira PIC, Carvalho AGC, Barros MFA, Lima MC. Fatores associados ao estado nutricional em crianças de creches públicas do município de Recife, PE, Brasil. Rev Bras Epidemiol 2013; 16(2):502-512.

23. Dallabona A, Cabral SC, Holfeman DA. Variáveis infantis e maternas associadas à presença de sobrepeso em crianças de creches. Rev Paul Pediatr 2010; 28(4):304-313.

24. Motta MEFA, Silva GAP, Araújo OC, Lira PI, Lima MC. $\mathrm{O}$ peso ao nascer influencia o estado nutricional ao final do primeiro ano de vida? J Pediatr 2005; 81(5):377382.

25. Rossi CE, Vasconcelos FAG. Peso ao nascer e obesidade em crianças e adolescentes: uma revisão sistemática. Rev Bras Epidemiol 2010; 13(2):246-258.

26. Tourinho AB, Reis LBSM. Peso ao Nascer: Uma Abordagem Nutricional. Com Ciências Saúde 2013; 22(4):19-30.

27. Niehues JR, Gonzales AI, Lemos RR, Bezerra PP, Hass P. Prevalence of Overweight and Obesity in Children and Adolescents from the Age Range of 2 to 19 Years Old in Brazil. Int J Pediatr 2014; 2014:1-7.

28. Magalhães TCA, Pires CN, Firmino HH, Franceschini SCC, Sant'Ana LFR. Aspectos relacionados à saúde infantil e principais alterações nutricionais em crianças brasileiras: revisão de literatura. Cad Saude Colet 2009; 17(2):433-452.

29. Figueroa Pedraza D, Menezes TN. Caracterização dos estudos de avaliação antropométrica de crianças pré -escolares brasileiras assistidas em creches. Rev Paul Pediatr 2016; 34(2):216-224.
30. Zöllner CC, Fisberg RM. Estado nutricional e sua relação com fatores biológicos, sociais e demográficos de crianças assistidas em creches da prefeitura do município de São Paulo. Rev Bras Saúde Matern Infant 2006; 6(3):319-328.

31. Barros FC, Victora CG, Scherpbier R, Gwatkin D. Socioeconomic inequities in the health and nutrition of children in low/middle income countries. Rev Saude Publica 2010; 44(1):1-16.

32. Assis AMO, Barreto ML, Oliveira MLP, Oliveira VA, Prado MS, Gomes GSS, Conceição Pinheiro SM, Dos Santos NS, Ribeiro da Silva RC, Ramos Sampaio L, Pacheco Santos LM. Determinants of mild-to-moderate malnutrition in preschoolers in an urban area of Northeastern Brazil: a hierarchical approach. Public Health Nut 2007; 11(4):387-394.

33. Barbosa RMS, Salles-Costa R, Soares EA. Estado nutricional de crianças atendidas em uma creche filantrópica: estudo longitudinal de 6 meses. Aliment Nutr 2007; 18(2):215-221.

34. Biscegli TS, Polis LB, Santos LM, Vicentin M. Avaliação do estado nutricional e prevalência da carência de ferro em crianças frequentadoras de uma creche. Rev Paul Pediatr 2007; 24(4):323-329.

35. Flores-Huerta $\mathrm{S}$, Klünder-Klünder M, Muños-Hernández O. Physical growth and nutritional status of Mexican infants from newborn to two years of age. Salud Publica 2012; 54(S1):82-89.

36. Oyhenart EE, Torres MF, Quintero FA, Luis MA, Cesani MF, Zucchi M, Orden AB. Estado nutricional y composición corporal de niños pobres residentes en barrios periféricos de La Plata, Argentina. Rev Panam Salud Publica 2007; 22(3):194-201.

37. Del Real SI, Jaeger AS, Barón MA, Díaz N, Solano L, Velásquez E, López J. Estado nutricional en niños preescolares que asisten a un jardín de infancia público en Valencia, Venezuela. ALAN 2007; 57(3):248-254.

38. Oliveira FCC, Cotta RMM, Sant'Ana LFR, Rocha SEP, Franceschini SCC. Programa Bolsa Família e estado nutricional infantil: desafios estratégicos. Cien Saude Colet 2011; 16(7):3307-3316.

39. Damaceno RJP, Martins PA, Devincenzi MU. Estado nutricional de crianças atendidas na rede pública de saúde do município de Santos. Rev Paul Pediatr 2009; 27(2):139-147.

40. Barroso GS, Sichiri R, Salles-Costa R. Fatores associados ao déficit nutricional em crianças residentes em uma área de prevalência elevada de insegurança alimentar. Rev Bras Epidemiol 2008; 11(3):484-494.

41. Moreira MA, Cabral PC, Ferreira HS, Lira PIC. Excesso de peso e fatores associados em crianças da região nordeste do Brasil. J Pediatr 2012; 88(4):347-352.

42. Saldiva SRDM, Silva, LFF, Saldiva PHN. Avaliação antropométrica e consumo alimentar em crianças menores de cinco anos residentes em um município da região do semiárido nordestino com cobertura parcial do programa bolsa família. Rev Nutr 2010; 23(2):221-229. 
43. Menezes RCE, Lira PIC, Leal VS, Oliveira JS, Santana SCS, Sequeira LAS, Rissin A, Batista Filho M. Determinantes do déficit estatural em menores de cinco anos no Estado de Pernambuco. Rev Saude Publica 2011; 45(6):1079-1087.

44. Barata RB. Epidemiologia e políticas públicas. Rev Bras Epidemiol 2013; 16(1):3-17.

45. Morais DC, Dutra LV, Franceschini SCC, Priore SE. Insegurança alimentar e indicadores antropométricos, dietéticos e sociais em estudos brasileiros: uma revisão sistemática. Cien Saude Colet 2014; 19(5):1475-1488.

46. Rinaldi AEM, Pereira AF, Macedo CS, Mota JF, Burini RC. Contribuições das práticas alimentares e inatividade física para o excesso de peso infantil. Rev Paul Pedia$\operatorname{tr} 2008 ; 26(3): 271-277$.

Artigo apresentado em 07/03/2015 Aprovado em 22/08/2015

Versão final apresentada em 24/08/2015 p. 2219

\section{onde se lê:}

Dixis Figueroa Pedraza ${ }^{1}$

Maria Monica de Oliveira ${ }^{2}$

Ana Carolina Dantas Rocha ${ }^{3}$

Erika Morganna Neves de Araujo ${ }^{1}$

leia-se:

Dixis Figueroa Pedraza ${ }^{1}$

Maria Monica de Oliveira ${ }^{2}$

Maria Vera Lúcia Moreira Leitão Cardoso ${ }^{3}$

Erika Morganna Neves de Araujo ${ }^{1}$

Ana Carolina Dantas Rocha ${ }^{3}$

p. 2230

onde se lê:

D Figueroa Pedraza participou da elaboração do projeto, concepção do artigo, revisão bibliográfica, análise e interpretação dos dados, redação e aprovação do artigo. MM Oliveira participou da revisão bibliográfica, análise e interpretação dos dados, redação e aprovação do artigo. ACD Rocha e EMN Araújo participaram da interpretação dos dados, redação e aprovação do artigo.

\section{leia-se:}

D Figueroa Pedraza participou da elaboração do projeto, concepção do artigo, revisão bibliográfica, análise e interpretação dos dados, redação e aprovação do artigo. MM Oliveira participou da revisão bibliográfica, análise e interpretação dos dados, redação e aprovação do artigo. MVLML Cardoso participou da revisão crítica e aprovação da versão final. ACD Rocha e EMN Araújo participaram da interpretação dos dados, redação e aprovação do artigo. 